\title{
Salivary Glands and Periodontal Changes in a Population of Sjögren's and Sicca Syndrome Treated by Pilocarpine: A Pilot Study
}

\author{
Thibaud Depinoy - Alain Saraux - Jacques-Olivier Pers · \\ Sylvie Boisramé · Divi Cornec · Thierry Marhadour · Dewi Guellec • \\ Valérie Devauchelle-Pensec · Luc Bressollette · Sandrine Jousse-Joulin
}

Received: October 13, 2020 / Accepted: November 26, 2020 / Published online: December 17, 2020

(C) The Author(s) 2020

\section{ABSTRACT}

Introduction: Oral administration of pilocarpine enhances salivary flow in sicca patients but its effect upstream on ultrasound (US) of salivary glands (SG) and downstream on periodontium remain unknown.

Methods: Sicca patients were prospectively included. Echostructural and vascularization of SG were assessed using B mode and pulsed

Supplementary Information The online version contains supplementary material available at https:// doi.org/10.1007/s40744-020-00263-y.

T. Depinoy · A. Saraux · D. Cornec $\cdot$ T. Marhadour · D. Guellec · V. Devauchelle-Pensec .

S. Jousse-Joulin $(\bowtie)$

Rheumatology Department, CHU de Brest, Brest, France

e-mail: sandrine.jousse-joulin@chu-brest.fr

A. Saraux · J.-O. Pers · D. Cornec .

V. Devauchelle-Pensec $\cdot$ S. Jousse-Joulin

UMR1227, Lymphocytes B Et Autoimmunité, Univ

Brest, INSERM, LabEx IGO, Brest, France

J.-O. Pers · S. Boisramé

Dental and Oral Surgery Unit, CHU de Brest Et Univ Brest, Brest, France

D. Guellec

Rheumatology Department, and INSERM CIC 1412, CHU de Brest, Brest, France

L. Bressollette

Ultrasound Unit Department, CHU de Brest, Brest, France
Doppler (USPD). Vascularization of SG was measured using resistive index (RI) before and after stimulation by lemon juice. Echostructure (measure of glandular length in $\mathrm{cm}^{2}$, evaluation of parotid and submandibular glands parenchymal abnormalities) was assessed at baseline (M0) and after 3 months (M3) of treatment with pilocarpine. A dental consultation was performed at M0 and M3 to evaluate changes in unstimulated salivary flow (USSF), stimulated salivary flow (SSF), and periodontal parameters such as modified gingival index (Lobene), plaque index (Silness), bleeding index, pocket depth, and $\mathrm{pH}$.

Results: Nineteen patients were included but only 11 received pilocarpine treatment for 3 months, as six stopped pilocarpine due to side effects and two were excluded for other causes. Among the 11 patients who completed the 3-month follow-up, five had primary Sjögren's syndrome according to the American-European's classification criteria. As expected, statistical differences were found concerning SSF $(p=0.018)$ and USSF $(p=0.027)$ between M0 and M3 while no statistical change in both SG echostructure and vascularization or periodontal evaluation was shown.

Conclusions: Pilocarpine improved SSF and USSF measurements in sicca syndrome but no ultrasonography of major salivary glands (SGUS) structural and vascular changes were detected as well as periodontal evaluation. 
Keywords: Pilocarpine; Salivary glands; Sjögren's syndrome; Ultrasonography

\section{Key Summary Points}

Evaluating the impact of treatment in patients with Sjögren's syndrome is still challenging.

This is the first study showing how to evaluate treatment using ultrasonography of major salivary glands in primary Sjögren's syndrome.

Pilocarpine improves salivary flow but does not change echostructural and vascularization of salivary glands in our pilot study. Unfortunately, a large number of patients were excluded due to poor treatment tolerance.

A larger population study and maybe another treatment is needed to evaluate echostructural and vascularization ultrasound changes in salivary glands.

\section{DIGITAL FEATURES}

This article is published with digital features, including a summary slide, to facilitate understanding of the article. To view digital features for this article go to https://doi.org/10.6084/ m9.figshare.13285970.

\section{INTRODUCTION}

Sicca syndrome refers to the dryness of the eyes and mouth. The main causes of this syndrome are ageing, medication, Sjögren's syndrome, cervicofacial radiotherapy, consequences of surgical intervention as well as other systemic pathologies such as diabetes and sarcoidosis [1]. A frequent symptom is xerostomia, which is a subjective feeling of oral dryness affecting about $10 \%$ of individuals. Sicca symptoms are often associated with fatigue, anxiety, depression, and quality-of-life impairments [2].

Recently, some imaging techniques have been developed to assess SG and particularly ultrasound in sicca syndrome and in primary Sjögren's syndrome (pSS) patients. However, ultrasonography of major salivary glands (SGUS) was poorly studied in sicca syndrome except for pSS. Takagi et al. found minimal pathological ultrasonographic findings on patients' salivary glands (SG) with xerostomia without pSS (140 patients with grade 0 among the SGUS of the 149 patients) [3]. Besides, Doppler has not yet been studied in patients with sicca syndrome without pSS. SGUS has been developed in the field of pSS and showed good correlation with histopathology of minor salivary glands, serology, and salivary gland function [4]. Correlations between clinical findings such as dry mouth [5] and impaired salivary flow with abnormalities in the submandibular glands blood flow was seen [6]. Until now, to evaluate xerostomia, physicians used unstimulated salivary flow (USSF) or stimulated salivary flow (SSF), which consist in collecting the amount of saliva produced by SG before and after stimulation. Indeed, the salivary flow is variable over time and depends on multiple factors such as the vascularization of SG. SG has an important vascular supply and could explain the salivary flow variation during stimulation. The study of vascularization could be an interesting tool to evaluate the salivary inflammation state. Some authors [7] have shown the interest to assess vascularization using pulsed color Doppler ultrasonography (USPD). They showed in pSS patients a decreased resistive index (RI) compared to controls and suggested that blood inflow responses to secretory stimulation may be defective in SG from pSS patients. Decrease of RI with a cutoff of less than 0.8 as described by Jousse-Joulin et al. [8] seems to correspond to the hyperemia status of SG in pSS patients. However, there are no publications describing the vascular status of sicca syndrome patients.

There are several symptomatic treatments for xerostomia, teaming up education, environment modification, elimination of contingent offending drugs, artificial tears, sialogogue 
medications (cholinergic agents) like cevimeline or pilocarpine and treatments for complications [9]. Oral administration of pilocarpine is effective for treating xerostomia and is the only validated treatment in patients with sicca syndrome [9] at the dosage of $5 \mathrm{mg}$ four times daily and is for some authors well tolerated [10] despite some adverse effect such as sweating [11]. However, in other studies, pilocarpine caused a high incidence of unacceptable adverse effects with a tolerated rate of $47 \%$ [12]. Pilocarpine is also effective in the treatment of xerostomia from radiation therapy and graft-versus-host disease $[13,14]$. This treatment is well known to be more effective than artificial saliva for enhancing salivary and lachrymal secretion in patients with pSS [15].

To date, pilocarpine's impact on SG is still assessed by SSF or USSF. Xerostomia is a subjective clinical symptom and even if the evaluation of disease activity is a main issue in sicca syndrome, few studies published data on imaging technique and treatment management in this syndrome. The use of SGUS in sicca syndrome except for pSS patients is not yet spread. However, evaluation of the echostructural changes and vascularization of major SG could be an interesting marker and ultrasound has not been yet validated in this condition.

To respond to this question, we conducted a pilot study (ECHOpilo study). The main objective was to evaluate ultrasound SG echostructure and vascular changes in sicca patients treated during 3 months by pilocarpine. Secondary objectives were to assess the dental and periodontal status of patients with xerostomia before and after 3 months treatment by pilocarpine.

\section{METHODS}

\section{Study Population}

Patients with sicca complaints were recruited by the odontology and oral surgery department and addressed to the ultrasound department to have an ultrasound examination. A screening visit was done at the ultrasound department to check vascular SG status and if inclusion criteria was fulfilled, the patients were included. Patients were recruited from October 2012 to November 2014 (30 patients a year). They were referred for exploration of a dry syndrome or suspicion of pSS.

Criteria for inclusion were xerostomia with or without pSS with pathological PDUS described as having a pathological RI $(\mathrm{RI}<0.8)$ with potential for salivary gland stimulation confirmed by objective measurement of salivary flow before and after stimulation by lemon juice with indication for treatment with pilocarpine (USSF less than $0.1 \mathrm{ml} / \mathrm{min}$ and less than the SSF).

Non-inclusion criteria were contraindication to pilocarpine (hypersensibility, asthma, glaucoma, iridocyclitis), medicated xerostomia, recent surgery $(<1$ month) pregnancy, HIV- or HBV-positive patients and treatment by corticosteroids or immunosuppressive treatments.

All patients gave written consent to the study during the dental consultation. The study obtained a favorable opinion from the committee for the protection of persons Ouest VI (16.08.2012) (2012-A00658-35) and fulfils compliance with the Declaration of Helsinki.

\section{Treatment Protocol and Evaluation of Treatment Efficacy}

Salivary glands were evaluated in B mode and pulsed Doppler. Inclusion criteria were based on patients with an abnormal vascular SG status (i.e., RI < 0.8) in order to observe a change with pilocarpine treatment defined as follows: decrease of the hyperemia SG status with a RI $>0.8$ or increase of hyperemia SG status with a RI $<0.8$.

After inclusion, patients received pilocarpine at $4 \mathrm{mg}$ four times daily during 3 months. At day 8 , a phone appointment was made to evaluate the pilocarpine tolerance.

At inclusion (M0) and after 3 months of treatment (M3), all patients underwent a standardized protocol evaluation including a clinical evaluation, a SGUS examination described as follows: measure of glandular length in $\mathrm{cm}^{2}$, SG evaluation (two parotids and two submandibular glands) parenchymal echostructure in B 
mode and study of SG vascularization response by measuring RI before and after stimulation by lemon juice), as well as a periodontal examination in the same day. Tolerance and observance to pilocarpine were also evaluated at M3 with the same standardized protocol examination.

\section{Salivary-Gland Ultrasonography (SGUS)}

The ultrasound device used was the IU 22 Philips Medical Systems, Andover, MA, USA, coupled with USPD high linear frequency probe $12 \mathrm{MHz}$.

All USPD examinations were assessed by the same ultrasonographer (SJJ). A standardized protocol was used as follows: patient seated in a semi-sitting position with the head slightly turned to the right side in order to see the screen of the machine and after the left side was evaluated. The procedure of USPD was the same as described by Carotti et al. [16]. The parenchymal echostructure of the four major salivary glands and the vascularization of the left parotid gland were evaluated. The M3 SGUS evaluation was blinded. To evaluate the echostructural parenchymal abnormalities, we used two grading systems. A previous grading system was described as follows, where grade 0 indicates a normal homogeneous gland; grade 1 small hypoechoic areas; grade 2 multiple hypoechoic areas less than $2 \mathrm{~mm}$ in size; grade 3 multiple hypoechoic areas $2-6 \mathrm{~mm}$ in size; and grade 4 multiple hypoechoic areas larger than $6 \mathrm{~mm}[5,17]$. The new semi-quantitative scoring system recently published by the OMERACT SGUS group is defined as grade 0 if completely normal; grade 1 , mild inhomogeneity without anechoic or hypoechoic areas and hyperechogenic bands; grade 2, moderate inhomogeneity with focal anechoic or hypoechoic areas; grade 3, severe inhomogeneity with diffuse an-/hypoechoic areas occupying the entire gland [18]. During the screening period, the OMERACT scoring system did not exist and we decided to apply it by reading static images from this pilot study.

The grade's sum of the parotid (PG) and submandibular glands (SMG) for each patient was performed, and a total grade for the ultrasound glandular echo-structure ranged from 0 to 12 . We considered that this grade was pathological if there were two glands with a grade greater than two, so a total sum of four glands greater than eight.

The procedure to evaluate the vascularization of the salivary glands was as follows: we assessed the vascularization of the left parotid gland at the level of the transverse facial artery (branch of the external carotid artery). We chose arbitrarily the left parotid gland for the comfort of the patient who could see the screen and for the comfort of the ultrasonographer. Indeed, we chose one gland to study the vascularization because by using stimulation by lemon we create an increase of the vascularization in each gland, which could influence the results of the other three glands if we use lemon again, which can lead to false results concerning the vascularization of the other glands. First, we used color Doppler to identify the transverse facial artery, and secondly we used pulsed Doppler color for evaluating the measurement of the RI. We recorded the Doppler waveform and measured the RI automatically (Fig. 1). We did the measurement before and after lemon stimulation. For the lemon stimulation, we used a syringe with $3 \mathrm{ml}$ of lemon juice, and we injected the syringe in the mouth of the patient. Just after this, we recorded the RI, and $1 \mathrm{~min}$ later after swallowing. We defined a cutoff of measurement for abnormal RI less than 0.8. [8]

\section{Dental Consultation}

The periodontal consultation was done by the same clinician (SB). A salivary flow was measured by a saliva pump connected to a swab during $10 \mathrm{~min}$ to calculate the USSF and then during 5 min after chewing a paraffin block to calculate the SSF. For the saliva $\mathrm{pH}$ value, all patients moisturized a $\mathrm{pH}$ test strip for $10 \mathrm{~s}$. Then, the color of the strip was checked and compared to the testing chart available in the package ( $\mathrm{pH}$ indicator paper by Merck ${ }^{\circledR}$ ).

Six teeth were selected using Ramfjord's guidelines [19]: the maxillary right first molar, the left central incisor, the left first premolar, the mandibular right central incisor, the left 


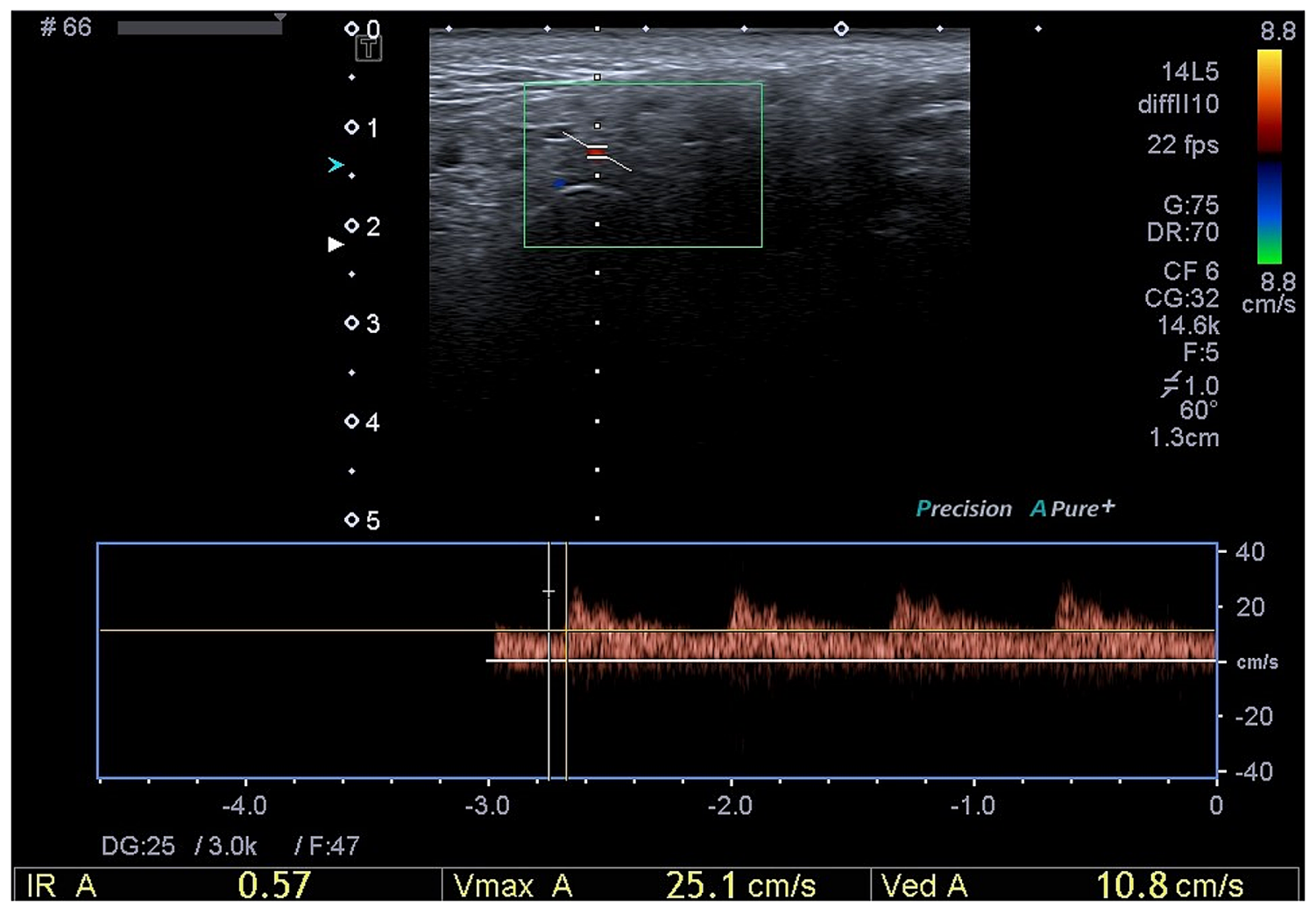

Fig. 1 Doppler waveform analysis of arterial supply in parotid gland (Pathologic Resistive Index: 0.58). The Doppler waveform analysis shows a decrease of the systolic

first molar, and the right first premolar. When an individual had a missing tooth, the distal tooth on the corresponding side was assessed. As previously described [20], the plaque index (Sillness and Loe index, [21]), gingival index (Lobene index, [22]) and papillary bleeding index were expressed as scores. For each index, the patient's final score was obtained by summing the most elevated index for each of the six teeth $[23,24]$. This gave a possible score range of 0-24 for the gingival index and 0-18 for the plaque and papillary bleeding indices. Pocket depth was defined as the distance in $\mathrm{mm}$ from the most coronal portion of the free gingival margin to the most apical penetration of the constant pressure probe. All measures were performed at the buccal, lingual, mesio-buccal, mesio-lingual, disto-buccal, and disto-lingual sides of each tooth. The maximum value was noted for each of the six teeth, and scores are presented as the mean of the six values. peak, which explains the low resistive index (IR: 0.57) result in pSS patients

\section{Statistical Analysis}

Our study was an exploratory pilot trial. As the primary endpoint was ultrasound evolution after a 3-month follow-up, statistical analysis was performed only on patients with ultrasonography results treated for 3 months by pilocarpine and evaluated using ultrasound at inclusion and after a 3-month follow-up. We aimed to include 30 patients in order to obtain 15 couples of sonography after/before treatment. We divided our sicca syndrome population into two subgroups: non-pSS and pSS patients.

As the study was pilot, with an objective of feasibility, we did not calculate a sample size but considered that if the improvement is statistically significant on clinical signs and not using ultrasound, the interest is modest to evaluate evolution. 
Data were analyzed using IBM Statistical Package for Social Sciences (SPSS) software version 23.0. (IBM Corporation, Armonk, NY, USA). Quantitative variables are presented as the mean \pm standard deviation, but as the number of patients was low, we added the minimum and maximum. For comparison before and after treatment, we used the Wilcoxon' paired test. The threshold of statistical significance was set at $p<0.05$.

\section{RESULTS}

\section{Description of the Study Population}

Among the 55 patients screened during the inclusion period (Fig. 2), we included 19 female patients (other patients did not meet the inclusion criteria; particularly they had a resistive index greater than 0.8); 11 patients (five met AECG criteria for pSS, six had non-pSS sicca) had completed the study (mean age, 55), whereas eight did not (mean age, 61; five discontinued treatment due to side effects, one did not take treatment due to non-reimbursement, one did not come to the consultation at M3 and one discontinued treatment due to chronic bilateral parotid sialodochitis).

\section{Results of the Different Dental Measurements Between M0 and M3}

Table 1 shows the different results of periodontal measurements at M0 and M3 in the total population of our patients. The means of all periodontal parameters (modified gingival, plaque, bleeding indices, and pocket depth) were not modified at M3 compared to M0. Saliva $\mathrm{pH}$ seemed to be lower at M0 compared to M3 $(p=0.06)$, but the difference was not statistically significant. We found that USF and SSF mean were greater at M3 $(p=027$ for USF and $p=0.018$ for SSF) with a statistically significant difference.

\section{Results of SGUS Measurement Surface at M0 and M3}

We found no statistical differences concerning parotid and submandibular gland surface between M0 and M3.

\section{Results of SGUS Parenchymal Echostructure and Vascularization (RI) in the Population}

Table 2 shows the different results of SGUS echostructural grading and vascularization at $\mathrm{M} 0$ and $\mathrm{M} 3$ in the total population. Concerning the SGUS echostructural changes, the mean SG grades using the previous scoring system seemed quite higher at the PG level than SMG, whatever M0 or M3 nevertheless to nonpathological values according to previous grading. The sum of the four SGUS grades did not change at M3 $(p=0.450)$. The mean SG grades using the new OMERACT scoring system seemed quite higher at the SMG level at M3, but with no statistical differences, and the sum of the four SGUS grades did not change at M3 $(p=0.167)$.

The means of the RI were also similar at M0 versus M3 before, immediately after and $1 \mathrm{~min}$ after stimulation with lemon juice. The variations of the RI with stimulation by lemon juice were not so different between M0 and M3 $(p=0.799$ for the comparison just after lemon juice stimulation) (Table 2). Finally, we found no significant difference in our sicca syndrome population study before and after 3 months of treatment with pilocarpine concerning SGUS parameters.

Concerning the analysis of our population into two subgroups (pSS and non-pSS), we did not perform statistical analysis because of the small number of patients. However, the means and standard deviations of the different parameters of the patient subgroups are presented in supplementary files 1 and 2 . In these supplementary files, we observed that patients with pSS have more pathological periodontal indexes [22], lower SSF, USSF, and higher SGUS grades compared to non-pSS patients. Besides, concerning vascularization of SG in these two 


\section{5 screened patients from October}

2012 to November 2014

\section{6 patients not meeting inclusion criteria (RI>à 0.8)}

19 patients fulfilling inclusion criteria (Resistive Index $<0.8$ ) for analysis
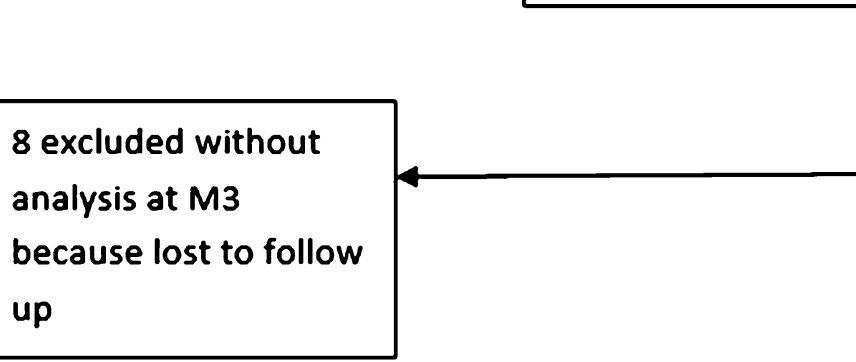

Fig. 2 Flowchart of the screening patients from October 2012 and November 2014

subgroups, we did not find any difference in terms of RI.

\section{DISCUSSION}

Patients with sicca syndrome represent a heterogeneous population with impaired salivary gland function without characteristic gland changes using ultrasonography. This could explain the lack of difference found between the sum of the grades of SG at M0 and
M3. Consequently, the grade improvement was difficult to demonstrate. In contrast, the sum of grades for the pSS patients seemed greater than in the non-pSS population. These preliminary results highlight the echostructural difference between our two sub-groups. The lack of SGUS change difference between M0 and M3 could also be explained by the small number of patients who completed the study (five out of 19 patients who stopped treatment due to side effects) and the short time of follow-up to evaluate treatment response. 
Table 1 Results of the different dental measurement at M0 and M3 in sicca syndrome population

\begin{tabular}{llcc}
\hline & $\begin{array}{l}\text { M0 (baseline) mean } \pm \text { SD } \\
(\text { min-max })\end{array}$ & $\begin{array}{l}\text { M3 mean } \pm \text { SD } \\
(\text { min-max })\end{array}$ & $p$ value \\
\hline Modified Gingival Index & $4.90 \pm 3.99(0-13)$ & $5.89 \pm 4.05(0-11)$ & 0.526 \\
Plaque Index & $6.90 \pm 3.87(3-12)$ & $6.78 \pm 3.35(0-10)$ & 0.674 \\
Bleeding Index & $3.70 \pm 3.27(0-10)$ & $5.56 \pm 3.5(0-11)$ & 0.201 \\
Pocket Depth (mm) & $4.56 \pm 7.09(0-19)$ & $4.89 \pm 5.11(0-14)$ & 0.686 \\
Stimulated Salivary Flow (SSF) (ml/min) & $0.50 \pm 0.41(0-1.25)$ & $0.59 \pm 0.47(0.01-1.50)$ & 0.018 \\
$\begin{array}{l}\text { Unstimulated Salivary Flow (USSF) (ml/ } \\
\text { min) }\end{array}$ & $0.18 \pm 0.17(0-0.50)$ & $0.28 \pm 0.18(0-0.60)$ & 0.027 \\
pH & $6.65 \pm 0.85(5.5-8)$ & & 0.068 \\
\hline
\end{tabular}

$S D$ standard deviation, min minimum, max maximum

Few studies have evaluated the impact of treatment on SGUS changes. Takagi showed that salivary gland US grading (sum of grades of four glands according to De Vita et al.) [3, 6] was associated with the response of xerostomia treatment (saline or corticosteroid irrigation, cevimeline gargle, oral cevimeline, oral pilocarpine, Kampō medicine, or oral corticosteroids) in patients with pSS, with a treatment duration of 10-11 months. The baseline salivary gland US score before treatment was the most important factor for predicting a bad response to pilocarpine in pSS patients, whereas the results were not statistically significant in patients with xerostomia without pSS. Indeed, patients with a lower baseline SGUS score had a greater increase in salivary flow after pilocarpine in the case of pSS. Unfortunately, the SSF was not studied in this study nor the difference of SGUS score before and after treatment [3].

To date, ultrasonography is emerging as a good method for objectively evaluating the salivary glands. SGUS implementation among the ACR/EULAR criteria increased sensitivity from 87.4 to $91.1 \%$ when physician diagnosis was the gold standard [24]. However, even if US is able to diagnose echostructural changes in pSS patients using reliable items as echogenicity and homogeneity $[8,25]$, these changes are not found in a sicca syndrome population which could explain our results. Some authors showed that SGUS changes should be evaluated by grey scale but only in pSS patients $[26,27]$.

Until now, there have been few studies that have evaluated the impact of treatment in salivary glands using vascularization item $[8,24]$. In our pilot study, we decided to study the impact of pilocarpine on the SG vascularization in sicca syndrome. Carotti et al. [16] and Martinoli et al. [7] have already shown that USPD had a diagnostic interest in patients with pSS with a lower amplitude of variation of RI in these patients and a diastolic aspect of the Doppler waveform. In these two studies, the authors showed that pSS patients had a lower RI and a poorer response to lemon juice stimulation as described by Chikui et al. in 2000 [6].

Though no comparison had been done in a sicca syndrome population, Luciano showed that SGUS is a good tool to differentiate patients with pSS from patients with undifferentiated connective tissue disease associated with sicca syndrome [26]. We chose the RI as a US marker of inflammation because it might reflect the inflammatory state of soft tissue. RI is defined as the ratio between systolic velocity and the diastolic velocity on systolic velocity [8]. The study of vascularization in salivary glands is not yet assessed in routine practice, but some studies $[6,7]$ have shown that RI decreases in the pSS population versus healthy controls. However, Carotti showed in patients with and without 
Table 2 Results of ultrasound grading and resistive index (RI) in the population at baseline and M3

\begin{tabular}{|c|c|c|c|}
\hline & $\begin{array}{l}\text { Mo (baseline) mean } \pm \text { SD } \\
(\min -\max )\end{array}$ & $\begin{array}{l}\mathrm{M} 3 \text { mean } \pm \mathrm{SD} \\
(\min -\max )\end{array}$ & $p$ value \\
\hline Left PG length $(\mathrm{cm})$ & $4.66 \pm 0.94(3.58-6.41)$ & $5.42 \pm 1.07(3.23-6.99)$ & 0.260 \\
\hline Right PG length $(\mathrm{cm})$ & $4.51 \pm 1.07(2.86-5.92)$ & $5.08 \pm 1.20(3.13-6.86)$ & 0.327 \\
\hline Left SMG length $(\mathrm{cm})$ & $2.98 \pm 0,78(1.48-4.29)$ & $2.84 \pm 0.67(2.00-4.45)$ & 0.213 \\
\hline Right SMG length $(\mathrm{cm})$ & $2.78 \pm 0.90(1.46-4.27)$ & $3.09 \pm 0.78(1.69-4.04)$ & 0.123 \\
\hline Left PG grade* & $1.09 \pm 1.58(0-4)$ & $1.20 \pm 1.55(0-4)$ & 1 \\
\hline Right PG grade* & $1 \pm 1.61(0-4)$ & $1.10 \pm 1.60(0-4)$ & 1 \\
\hline Left SMG grade* & $0.73 \pm 1.35(0-4)$ & $0.82 \pm 1.47(0-4)$ & 0.317 \\
\hline Right SMG grade* & $0.73 \pm 1.35(0-4)$ & $0.91 \pm 1.45(0-4)$ & 0.157 \\
\hline Left PG OGrade & $1 \pm 1.247$ & $1 \pm 1.155$ & 1 \\
\hline Right PG OGrade & $0.9 \pm 1.287$ & $0.9 \pm 1.197$ & 1 \\
\hline Left SMG OGrade & $0.64 \pm 1.120$ & $0.73 \pm 1.272$ & 0.341 \\
\hline Right SMG OGrade & $0.64 \pm 1.120$ & $0.82 \pm 1.250$ & 0.167 \\
\hline Sum of four glands grades* & $4.33 \pm 6.12(0-16)$ & $4.56 \pm 6.23(0-16)$ & 0.450 \\
\hline Sum of four glands OGrades & $3.3 \pm 4.764$ & $3.6 \pm 4.76$ & 0.434 \\
\hline RI at baseline & $0.76 \pm 0.09(0.51-0.87)$ & $0.80 \pm 0.07(0.73-0.92)$ & 0.721 \\
\hline $\mathrm{RI}$ after stimulation & $0.73 \pm 0.15(0.35-0.90)$ & $0.76 \pm 0.10(0.61-0.94)$ & 0.953 \\
\hline RI 1 min after stimulation & $0.72 \pm 0.11(0.47-0.85)$ & $0.80 \pm 0.06(0.71-0.89)$ & 0.260 \\
\hline $\begin{array}{l}\text { Difference between RI after and before } \\
\text { stimulation }\end{array}$ & $-0.05 \pm 0.09(-0.19-0.05)$ & $\begin{aligned}- & 0.04 \pm 0.13 \\
& (-0.25-0.19)\end{aligned}$ & 0.799 \\
\hline
\end{tabular}

$S D$ standard deviation, min minimum, max maximum, $R I$ resistive index, $P G$ parotid gland, $S M G$ submandibular gland ${ }^{*}$ Grading system using previous scoring system [17]; Ograde: grading using new OMERACT scoring system

pSS that the variability of RI in salivary glands before and during lemon juice stimulation was not statistically significant in either pSS patients or controls with xerostomia [16].

We decided arbitrarily to measure RI at the left parotid gland for two reasons. The first is to give the possibility to our patients to see the screen, and the second is to evaluate correctly lemon and stimulation. We also chose the vascularization of the parotid gland because the majority of published studies have shown results only on submandibular glands [6]. Martinoli et al. studied vascularization on the three main salivary glands [7], and Chikui et al. performed RI measurements on the facial artery at the level of the submandibular gland [6]. Carotti et al. measured both the external carotid artery and the facial artery in the submandibular gland [16]. In future studies, it could be interesting to combine RI of the parotid and sub-mandibular glands to see some differences at each level. Regarding RI values, pilocarpine acted on muscarinic receptors of salivary gland vessels and therefore was an arterial vasodilator. Arterial vasodilation resulted in an increase of the diastolic velocity and therefore a decrease in the arterial RI. Lemon juice stimulation and pilocarpine had the same effect on the salivary 
gland vessels, causing vasodilation as shown by Martinoli et al. in 1994 [7].

We expected, above all, a better response of RI with lemon juice stimulation related to a better vasodilation of the vessels after treatment with pilocarpine, testifying to a better capacity of the salivary glands to adapt their vascularization and secretion to the food intake. We can suggest that the absence of difference found could be explained by the low number of patients or because pilocarpine had little effect on the resistance index after 3 months, a longer duration of treatment, or a novel treatment could be a perspective. Recently, SGUS vascularization changes in pSS patients treated by Rituximab [24] were evaluated using power Doppler, but unfortunately no statistical changes in vascularization were observed.

In the future, it might be interesting to evaluate the different vascularization approaches in sicca and Sjögren's syndrome population and compare the use and the interest of US combined with pulsed Doppler versus power Doppler [27], or with new super microvascular imaging [27]. The SGUS OMERACT group is working on a new vascular semi-quantitative scoring system in pSS patients and this work could permit having an evaluation of the inflammatory state in this population and to follow treated patients.

In our study, there was no improvement in periodontal indices at M3 for the modified gingival, plaque, and bleeding indices even if we were aware that 3 months should be too short to observe any change in dental status in our patients. Nonetheless, the mean pocket depth was pathological (>3 mm) whatever the subgroup of patients, pSS or non-pSS. These results are in accordance with the publication of Le Gall et al. in 2016 [23]. Whole saliva pH was also lower in pSS patients and associated with greater severity of periodontal disease.

We found a statistically significant increase in SSF and USSF after 3 months of pilocarpine. This increase was also found in the study of $\mathrm{Wu}$ in 2006 after 3 months of treatment [11], as well as in the recent study of Cifuentes (2018) who compared the use of artificial saliva versus pilocarpine in patients with pSS [15].
Although pilocarpine has an impact on salivary flow, this treatment is controversial regarding its tolerance. This parasympathetic agent increases salivary secretion by stimulating muscarinic M3 receptors, and the main reported side effects are sweating (with an incidence of greater than $50 \%$ in some studies), nausea, vomiting, diarrhea, headache, chest pain, wheezing, abdominal cramps, dizziness, palpitations, asthenia, chills, increased urinary frequency, and rhinitis in several studies [28, 29]. In a Japanese study published in 2009, the use of pilocarpine at $5 \mathrm{mg}$ three times daily lead to a good tolerance in only $47 \%$ of patients. The most common adverse effect was sweating, with an incidence of $64 \%$ in 36 patients [12]. On the contrary, in other studies, the treatment was better tolerated, with only $21.7 \%$ sweating due to pilocarpine [11]. In Noisaeh et al.'s study, there was $61 \%$ withdrawal of treatment with pilocarpine compared to $32 \%$ with cevimeline in 118 patients treated in total [30], but in 2013, an American pilot study described a slightly higher increment in saliva with pilocarpine compared to cevimeline. However, the difference was not statistically significant [31]. It might be interesting to study the modifications of the RI under cevimeline, which seems better tolerated. In consequence, the side effects due to pilocarpine explain our low recruitment of patients. In healthy subjects, a randomized double-blinded placebo-controlled study showed that a single rinse with $1-2 \%$ pilocarpine mouthwash significantly increased salivation without cardiovascular, visual, gastrointestinal, or behavioral side effects $[14,32]$, but improving salivary flow with mouthwash was not found in other studies, with similar efficacy of pilocarpine mouthwash and saline solution on salivary flow [32]. It would also be interesting to evaluate the response to other new treatments. However, we need to take into consideration that in our study, patients did not receive any corticosteroids or immunosuppressive treatments that could modify the response to treatment by pilocarpine.

Concerning the SSF and USSF, it is well known that pSS have severe oral and periodontal conditions [21, 33] due to the decrease of the salivary flow [34]. Unfortunately, in our 
study, we did not find any improvement of periodontal conditions, which was maybe due to a follow-up period that was too short.

The main limitations of our study were the low number of patients, the short follow-up time, and the monocentric design with only one expert. As a result, the small size of patients provides low power of our study allowing no definitive conclusion despite the absence of numerical differences on the primary endpoint. However, one of the highlights of this study is its prospective nature explaining the large number of excluded patients for side effects of treatment, and it is the first to study SG vascularization in sicca and Sjögren's syndrome as a new approach to evaluating the response to treatment.

\section{CONCLUSIONS}

To conclude, this pilot study is the first attempt to set forth preliminary results about response to treatment by means of SGUS. Pilocarpine improved SSF and USF measurements in our sicca syndrome population without any structural or vascular US changes.

\section{ACKNOWLEDGEMENTS}

We would like to thank all patients participating in the study.

Funding. No funding or sponsorship was received for this study or publication of this article.

Authorship. All named authors meet the International Committee of Medical Journal Editors (ICMJE) criteria for authorship for this article, take responsibility for the integrity of the work as a whole, and have given their approval for this version to be published.

Disclosures. Alain Saraux is an editorial board member for Rheumatology and Therapy, but has nothing else to disclose. None of the remaining authors (Thibaud Depinoy, JacquesOlivier Pers, Sylvie Boisramé, Divi Cornec,
Thierry Marhadour, Dewi Guellec, Valérie Devauchelle-Pensec, Luc Bressollette, Sandrine Jousse-Joulin) have anything to disclose.

Compliance with Ethics Guidelines. The study obtained an IRB approval committee for the protection of persons Ouest VI (16.08.2012) (2012-A00658-35) and fulfils compliance with the Declaration of Helsinki. All patients gave written consent to the study during the dental consultation.

Data Availability. The datasets generated during and/or analyzed during the current study are available from the corresponding author on reasonable request.

Open Access. This article is licensed under a Creative Commons Attribution-NonCommercial 4.0 International License, which permits any non-commercial use, sharing, adaptation, distribution and reproduction in any medium or format, as long as you give appropriate credit to the original author(s) and the source, provide a link to the Creative Commons licence, and indicate if changes were made. The images or other third party material in this article are included in the article's Creative Commons licence, unless indicated otherwise in a credit line to the material. If material is not included in the article's Creative Commons licence and your intended use is not permitted by statutory regulation or exceeds the permitted use, you will need to obtain permission directly from the copyright holder. To view a copy of this licence, visit http://creativecommons.org/licenses/bync/4.0/.

\section{REFERENCES}

1. Delli K, Spijkervet FK, Kroese FG, Bootsma H, Vissink A. Xerostomia. Monogr Oral Sci. 2014;24: 109-25.

2. Milin M, Cornec D, Chastaing $\mathrm{M}$, et al. Sicca symptoms are associated with similar fatigue, anxiety, depression, and quality-of-life impairments in patients with and without primary Sjögren's syndrome. Joint Bone Spine. 2016;83(6):681-5. 
3. Takagi Y, Sumi M, Nakamura H, Sato S, Kawakami A, Nakamura T. Salivary gland ultrasonography as a primary imaging tool for predicting efficacy of xerostomia treatment in patients with Sjögren's syndrome. Rheumatology (Oxford). 2016;55(2): 237-45.

4. Kim JW, Lee H, Park SH, Kim SK, Choe JY, Kim JK. Salivary gland ultrasonography findings are associated with clinical, histological, and serologic features of Sjögren's syndrome. Scand J Rheumatol. 2018;7:1-8.

5. De Vita S, Lorenzon G, Rossi G, Sabella M, Fossaluzza V. Salivary gland echography in primary and secondary Sjögren's syndrome. Clin Exp Rheumatol. 1992;10(4):351-6.

6. Chikui T, Yonetsu K, Izumi M, Eguchi K, Nakamura $\mathrm{T}$. Abnormal blood flow to the submandibular glands of patients with Sjögren's syndrome: doppler waveform analysis. J Rheumatol. 2000;27(5): 1222-8.

7. Martinoli C, Derchi LE, Solbiati L, Rizzatto G, Silvestri E, Giannoni M. Color Doppler sonography of salivary glands. AJR Am J Roentgenol. 1994;163(4): 933-41.

8. Jousse-Joulin S, Devauchelle-Pensec V, Morvan J, et al. Ultrasound assessment of salivary glands in patients with primary Sjögren's syndrome treated with rituximab: quantitative and Doppler waveform analysis. Biologics. 2007;1(3):311-9.

9. Saraux A, Pers JO, Devauchelle-Pensec V. Treatment of primary Sjögren syndrome. Nat Rev Rheumatol. 2016;12(8):456-71.

10. Vivino FB, Al-Hashimi I, Khan Z, et al. Pilocarpine tablets for the treatment of dry mouth and dry eye symptoms in patients with Sjögren syndrome: a randomized, placebo-controlled, fixed-dose, multicenter trial. P92-01 study group. Arch Intern Med. 1999;159(2):174-81.

11. Wu CH, Hsieh SC, Lee KL, Li KJ, Lu MC, Yu CL. Pilocarpine hydrochloride for the treatment of xerostomia in patients with Sjögren's syndrome in Taiwan-a double-blind placebo-controlled trial. J Formos Med Assoc. 2006;105(10):796-803.

12. Nakamura N, Sasano N, Yamashita H, et al. Oral pilocarpine ( $5 \mathrm{mg}$ t.i.d) used for xerostomia causes adverse effects in Japanese. Auris Nasus Larynx. 2009;36(3):310-3.

13. Berk L. Systemic pilocarpine for treatment of xerostomia. Expert Opin Drug Metab Toxicol. 2008;4(10):1333-40.
14. Tanigawa T, Yamashita J, Sato T, et al. Efficacy and safety of pilocarpine mouthwash in elderly patients with xerostomia. Spec Care Dentist. 2015;35(4): 164-9.

15. Cifuentes M, Del Barrio-Díaz P, et Vera-Kellet C. Pilocarpine and artificial saliva for the treatment of xerostomia and xerophthalmia of Sjögren's syndrome: a double-blind control trial. Br J Dermatol. 2018.

16. Carotti M, Salaffi F, Manganelli P, Argalia G. Ultrasonography and colour Doppler sonography of salivary glands in primary Sjögren's syndrome. Clin Rheumatol. 2001;20(3):213-9.

17. Cornec D, Jousse-Joulin S, Pers JO, et al. Contribution of salivary gland ultrasonography to the diagnosis of Sjögren's syndrome: toward new diagnostic criteria? Arthritis Rheum. 2013;65(1):216-25.

18. Jousse-Joulin S, D'Agostino MA, Nicolas C, et al. Video clip assessment of a salivary gland ultrasound scoring system in Sjögren's syndrome using consensual definitions: an OMERACT ultrasound working group reliability exercise. Ann Rheum Dis. 2019;78(7):967-73.

19. Ramfjord SP. The periodontal disease index (PDI). J Periodontol. 1967;38(Suppl):602-10. https://doi. org/10.1902/jop.1967.38.6.602.

20. Pers JO, Saraux A, Pierre R, Youinou P. Anti-TNFalpha immunotherapy is associated with increased gingival inflammation without clinical attachment loss in subjects with rheumatoid arthritis. J Periodontol. 2008;79(9):1645-51.

21. Silness J, Loe H. Periodontal disease in pregnancy. Ii. Correlation between oral hygiene and periodontal condition. Acta Odontol Scand. 1964;22: 121-35.

22. Lobene RR, Weatherford T, Ross NM, Lamm RA, Menaker L. A modified gingival index for use in clinical trials. Clin Prev Dent. 1986;8(1):3-6.

23. Le Gall M, Cornec D, Pers JO, et al. A prospective evaluation of dental and periodontal status in patients with suspected Sjögren's syndrome. Joint Bone Spine. 2016;83(2):235-6.

24. Le Goff M, Cornec D, Jousse-Joulin S, et al. Comparison of 2002 AECG and 2016 ACR/EULAR classification criteria and added value of salivary gland ultrasonography in a patient cohort with suspected primary Sjögren's syndrome. Arthritis Res Ther. 2017;19(1):269.

25. Fisher BA, Everett CC, Rout J, et al. Effect of rituximab on a salivary gland ultrasound score in primary Sjögren's syndrome: results of the TRACTISS 
randomised double-blind multicentre substudy. Ann Rheum Dis. 2018;77(3):412-6.

26. Luciano N, Baldini C, Tarantini G, et al. Ultrasonography of major salivary glands: a highly specific tool for distinguishing primary Sjögren's syndrome from undifferentiated connective tissue diseases. Rheumatology (Oxford). 2015;54(12): 2198-204.

27. Carotti M, Ciapetti A, Jousse-Joulin S, Salaffi F. Ultrasonography of the salivary glands: the role of grey-scale and colour/power Doppler. Clin Exp Rheumatol. 2014;32(1 Suppl 80):S61-70.

28. Hendrickson RG, Morocco AP, Greenberg MI. Pilocarpine toxicity and the treatment of xerostomia. J Emerg Med. 2004;26(4):429-32.

29. Zimmerman TJ, Wheeler TM. Miotics: side effects and ways to avoid them. Ophthalmology. 1982;89(1):76-80.
30. Noaiseh G, Baker JF, Vivino FB. Comparison of the discontinuation rates and side-effect profiles of pilocarpine and cevimeline for xerostomia in primary Sjögren's syndrome. Clin Exp Rheumatol. 2014;32(4):575-7.

31. Brimhall J, Jhaveri MA, Yepes JF. Efficacy of cevimeline vs. pilocarpine in the secretion of saliva: a pilot study. Spec Care Dentist. 2013;33(3):123-7.

32. Bernardi R, Perin C, Becker FL, et al. Effect of pilocarpine mouthwash on salivary flow. Braz J Med Biol Res. 2002;35(1):105-10.

33. Christensen LB, Petersen PE, Torn JJ, Schiodt M. Dental caries and dental health behavior of patients with primary Sjögren syndrome. Acta Odontol Scand. 2001;59(3):116-20.

34. Pijpe J, Kalk WW, Bootsma H, Spijkervet FK, Kallenberg CG, Vissink A. Progression of salivary gland dysfunction in patients with Sjögren's syndrome. Ann Rheum Dis. 2007;66(1):107-12. 